\title{
Adquisición De Datos De Magnitudes En Un Sistema Oleohidráulico Para Su Control Y Monitoreo En Un Laboratorio Remoto De Instrumentación Virtual De Código Abierto
}

\author{
Telmo Jesús Moreno Romero \\ Javier Enrique Orna Chávez
}

Docente - Investigador de la Facultad de Mecánica

Escuela Superior Politécnica de Chimborazo - Ecuador

Hugo Oswaldo Moreno Avilés

Docente - Investigador de la Facultad de Informática y Electrónica

Escuela Superior Politécnica de Chimborazo - Ecuador

\section{Álvaro Gonzalo Aguinaga Barragán}

Docente - Investigador de la Facultad de Mecánica

Escuela Politécnica Nacional - Ecuador

Nelson Gustavo Jara Cobos

Docente - Investigador de la Facultad de Mecánica

Universidad Politécnica Salesiana - Ecuador

\section{doi: 10.19044/esj.2017.v13n9p111 URL:http://dx.doi.org/10.19044/esj.2017.v13n9p111}

\begin{abstract}
The present research analyzes the state of the art of remote laboratories and their use in teaching and industry. In addition, the aim of this study is to develop a test bench to control and maintain, in optimal conditions, the operating parameters of a hydraulic oil system through an open source virtual platform. This equipment will analyze the behavior of an external gear pump which will enable us to make comparisons of mathematical models to predict the flow rate and compare it with that obtained in real time through the test bench. The analysis starts with a theoretical revision that is used for defining the variables to be controlled in the test bench. It is also used to propose a methodology that allows reaching the objectives raised.
\end{abstract}

Keywords: Virtual laboratory, mathematical model, external gears, pressure, flow 


\section{Resumen}

En este estudio se analiza el estado del arte de los laboratorios remotos y su uso en la enseñanza e industria. Además se pretende desarrollar un banco de pruebas para controlar y mantener en óptimas condiciones los parámetros de funcionamiento de un sistema oleo hidráulico por medio de una plataforma virtual de código abierto. En este equipo se analizará el comportamiento de una bomba de engranajes externos, para lo cual se realizarán comparaciones de modelos matemáticos que permitan predecir el caudal suministrado y así compararlo con el obtenido en tiempo real por medio del banco de pruebas. El análisis parte con una revisión teórica que permite definir las variables a ser monitoreadas y controladas en el banco de pruebas, para proponer una metodología que permita llegar a los objetivos planteados.

Palabras claves: Laboratorio virtual, modelo matemático, engranajes externos, presión, caudal

\section{Introducción}

La enseñanza mediante la educación virtual remota es cada vez más creciente debido a la facilidad que ofrece el internet para acceder desde cualquier parte del mundo a un laboratorio con el objetivo de programar, desarrollar el proceso, controlarlo y evaluarlo sin tener que estar en el sitio de trabajo. Los laboratorios remotos constituyen una herramienta muy importante para la academia y centros de investigación así como el control de equipos industriales. Por esta razón el laboratorio instrumental virtual remoto es una nueva alternativa para resolver necesidades para acceder a equipos físicos a distancia.

Estos laboratorios no reemplazan a los laboratorios tradicionales, más bien se complementan con éstos, así el estudiante de áreas técnicas como Ingeniería Mecánica requiere aún estar presente en la mayoría de laboratorios y talleres pero permite abaratar costos al permitir una mayor accesibilidad. Esta forma complementaria de educación genera una enseñanza constructivista ya que permite que el estudiante adquiera el conocimiento de forma autónoma y significativa, además él puede seguir adquiriendo conocimientos fuera de los "horarios normales". La posibilidad de experimentar en laboratorios desde cualquier lugar gracias al internet, representa una alternativa efectiva para que muchos estudiantes puedan utilizar en tiempo real y realizar todos los análisis e interpretaciones. 


\section{Problemática a solucionar}

Los laboratorios presenciales se siguen utilizando y los resultados de la pruebas son enviadas al computador para su análisis en función de los parámetros específicos. Este procedimiento además de ser costoso, requiere de mayor recurso humano para la enseñanza, limita la participación debido a la poca o inexistente disponibilidad de equipos para la experimentación, horarios fijos para el inicio y término de la práctica, lo que motiva que el estudiante no alcance a cumplir con todos los objetivos propuestos. Además, de todas maneras hay estudiantes que persisten en llenar los vacíos tratando de repetir las prácticas en otros espacios de tiempo disponibles para poder terminar las tareas asignadas, labor que regularmente no puede ser realizada debido a que el laboratorio se encuentra ocupado en otras clases, investigaciones o consultorías.

\section{Análisis comparativo del estado del arte}

El propósito de este trabajo es la construcción de un conjunto de experimentos automatizados que pueden ser controlados desde un ordenador remoto y crear un entorno web fácil de usar que permita el control remoto de estas tareas. Además, un número cada vez mayor de estudiantes en ciencias técnicas y/o naturales está en contraste con la capacidad de los laboratorios y el rápido desarrollo de las actividades informatizadas en la vida diaria requiere de un entrenamiento apropiado y acceso a los laboratorios las 24 horas a través del interfaz web desde cualquier ordenador (Petr Sládeka, 2011). Esta nueva forma de enseñanza se convierte en una educación a distancia, dadas la condiciones de la aplicación de la tecnología de la realidad virtual en un experimento remoto.

El laboratorio remoto se programa para el aprendizaje a distancia, como una nueva estrategia de formación de los estudiantes, acceso a los experimentos de laboratorio costosos, los experimentos se acompañan por una base teórica sobre la plataforma web. Dicho laboratorio debe interactuar entre el experimentador y el estudiante en el laboratorio a través de un ordenador personal conectado a Internet, es decir que los experimentos son reales y se ejecutan en el tiempo real con posibilidad de operar, medir y observar los experimentos a través de cámaras web. No es una simulación de experimentos o grabaciones de vídeo (Petr Sládeka, 2011). También se analiza la educación a distancia basada en la tecnología de la realidad virtual en 3-D enlazada a internet dentro de la teoría constructivista que explora, se trabaja en grupo y se realiza un estudio experimental. (Lei, 2012). Aquí, el alumno obtiene solo el estudio de los recursos de los experimentos virtuales en 3D (laboratorio virtual); además, ellos pueden comunicarse entre sí para llevar a cabo en colaboración experimentos y la interfaz del usuario puede proporcionar apoyos técnicos para todos los experimentos. El entorno virtual 
en 3D experimento (laboratorio virtual) es una rica base de conocimientos que representa los pensamientos de maestros y libros. Proporciona un conocimiento teórico y diseña los módulos de aprendizaje dirigidos a experimentos específicos (Lei, 2012).

Para que pueda desarrollarse el experimento en tiempo real, el sistema debe disponer de una serie de instrumentos de medida digitales, cuyas señales pueden ser enviadas a un servidor mediante un software a través de la web hacia cualquier computador que disponga de internet. Para ingresar el estudiante deberá disponer de un usuario y una clave personal. En este sentido en la Universidad del Táchira se ha desarrollado un experimento en el laboratorio de instrumentación electrónica, para lo cual los estudiantes requieren de una PC con una tarjeta de adquisición de datos. En este caso, los estudiantes realizan la instrumentación virtual desde sus casas y luego van al laboratorio a verificar el funcionamiento del mismo (Rugeles, 2002).

En cambio en la república Checa, el proceso se desarrolló en 400 escuelas, cuyos experimentos remotos pudieron ser implementados mediante un kit de software, El ISES kit consiste en una tarjeta de medida AD / DA (AD / DA convertidor), que se inserta en una ranura PCI (ISA) en el equipo que controla el experimento. Con estos recursos, los experimentos remotos se pueden acceder desde cualquier lugar en Internet (Casoli P., 2005) (Petr Sládeka, 2011).

Un nuevo enfoque para desarrollar prácticas a distancia para sistemas de ingeniería y laboratorios de control automático es basado en simulaciones fáciles de JavaScript (EJsS), Raspberry Pi y Node.js. Este se utiliza para crear un interfaz JavaScript y HTML5 que permite a los profesores y estudiantes parametrizar y observar el comportamiento de los controladores / sistemas en estudio a partir de los navegadores web de sus tabletas y teléfonos inteligentes. La Raspberry Pi, es una placa computadora de bajo costo, es la encargada de ejecutar 1) el programa de que cierra el bucle de control de la planta seleccionada y 2) el servidor web laboratorio JavaScript que aloja el interfaz de laboratorio y se comunica con el controlador (Cruz, 2015).

El análisis comparativo realizado se puede complementar mediante la incorporación de un Raspberry Pi conjuntamente con la tarjeta arduino para digitalizar la señales del banco de pruebas, luego enviarlas a través de internet a un servidor y de allí emitir hacia cualquier computador que mediante un usuario y clave podrán tener acceso a toda la información las pruebas programadas en los diferentes bancos de pruebas desarrollados en el presente proyecto. 


\section{Modelo matemático de bombas de engranajes externos}

Con la finalidad de comparar los resultados teóricos con los experimentales a continuación se analizan modelos matemáticos con los cuales se puede predecir el caudal suministrado por una bomba de engranajes externos. Las bombas de engranajes son ampliamente usadas debido a su alto rendimiento, elevada presión de descarga, alta fiabilidad y construcción compacta en general se usa bombas con engranajes externos (Huang $\mathrm{K}$, 2009), el principio de funcionamiento de este tipo de bombas se basa en un par de engranajes que provocan un cambio en el volumen entre los engranes y la cámara de la bomba lo que provoca un aumento de presión debido a la incompresibilidad del fluido.

El caudal de una bomba volumétrica es igual a su cilindrada por la velocidad angular del eje de la bomba, es decir (Svishchev A., 2015):

$$
Q_{p}=\frac{q_{p}}{2 \pi} w_{p}
$$

Dónde $\mathrm{w}_{\mathrm{p}}$ es la velocidad angular del eje, $\mathrm{q}_{\mathrm{p}}$ es la cilindrada de la bomba, la misma que depende del tipo de bomba, y su geometría. Para una bomba ideal la potencia transmitida al fluido es igual a la potencia mecánica por lo que igualando se puede obtener una expresión del torque:

$$
T=\frac{Q_{p} \cdot P}{w_{p}}
$$

El problema en la predicción del caudal suministrado por una bomba, es la modelación de la variación de la cilindrada la cual dependerá del tipo de bomba, geometría y del tipo de perfil del diente, en el caso de bombas de engranajes se presentan varios tipos de dientes (Martinez P., 2014) de los cuales principalmente se utilizan en la industria dientes de perfil envolvente y cicloidal.

A continuación se analizan 3 modelos propuestos para el cálculo de caudal de bombas de engranajes externos de perfil evolvente que son los que se utilizan ampliamente en la industria. Nakamura establece un modelo para el cálculo del caudal (Nagamura K., 2004), en el que enuncia que el volumen desplazado por revolución es igual a:

$$
V_{r}=\left(2 F-X_{\min }\right) z
$$

Donde $\mathrm{F}$ es el volumen del especio del diente $\mathrm{y}_{\mathrm{m}}$ min es el valor de volumen mínimo de separación y z es el número de dientes, para el caso de dientes de perfil envolventes:

$$
V_{r}=\frac{\pi b m^{2}}{2}\left((z+2+2(y-x))^{2}-(z+y)^{2}-\frac{\pi^{2}}{3} \cos ^{2} \alpha_{0}\right)
$$

Dónde $\mathrm{b}$ es el ancho de cara, $\mathrm{m}$ el módulo, $\mathrm{x}$ el coeficiente de addendum, y el coeficiente de dedendum y $\alpha_{0}$ el ángulo de presión. La expresión anterior es válida solo para dientes de perfil evolvente para el caso 
de otros tipos de perfil Nakamura propone una modificación a los coeficientes $\mathrm{x}, \mathrm{y}$.

Huang propone un modelo para representar el caudal de una bomba, el mismo que es igual a la diferencia entre el caudal volumétrico de entrada y el caudal volumétrico existente (Huang K, 2009):

$$
\dot{V}_{n}=\dot{V}_{0}-\dot{V}_{B}
$$

Debido a que el espesor de los engranajes es constante la misma puede salir del análisis, el caudal de área de entrada puede ser escrita:

$$
\dot{A}_{o}=\frac{1}{2} w\left(r_{a 1}^{2}+\frac{z_{1}}{z_{2}} r_{a 2}{ }^{2}\right)
$$

Se puede observar que el caudal de área de entrada depende de la velocidad angular de los engranajes (w), el número de dientes de los engranajes $\left(\mathrm{z}_{\mathrm{i}}\right)$, el radio de adendum de los engranajes $\left(\mathrm{r}_{\mathrm{ai}}\right)$. El caudal de área existente es igual a la sumatoria de los caudales en cada diente del engranaje, se puede expresar como:

$$
\begin{aligned}
\dot{A}_{B}=\frac{1}{2} w\left[\left(\frac{r_{1}{ }^{2}}{\left.\sqrt{r_{1}^{2}-r_{b 1}^{2}}\right)\left(r_{o p 1} \cdot \cos \beta_{1}-1\right)}\right.\right. \\
\left.\quad+\frac{z_{1}}{z_{2}}\left(\frac{r_{2}^{2}}{\sqrt{r_{2}^{2}-r_{b 2}^{2}}}\right)\left(r_{o p 2 \cdot} \cos \beta_{2}-1\right)\right]
\end{aligned}
$$

El caudal de área existente se expresa en función de la velocidad angular de los engranajes $(\mathrm{w})$, distancia radial de contacto $\left(\mathrm{r}_{\mathrm{i}}\right)$, radio de base del engranaje $\left(\mathrm{r}_{\mathrm{bi}}\right)$, radio primitivo de los engranajes $\left(\mathrm{r}_{\mathrm{opi}}\right)$, el ángulo de la línea de presión $\left(\beta_{\mathrm{i}}\right)$.

Casoli y Vacca presentan un modelo similar al de Nakamura considerando que el caudal de la bomba no es constante, éste depende del volumen en la descarga de la bomba (Casoli P., 2005) y se puede expresar como:

$$
Q_{p}=b_{w}\left(r_{a}^{2}-r_{w}^{2}-x_{p b}^{2}\right) w_{p}
$$

Donde $b_{\mathrm{w}}$ es el ancho del diente, $\mathrm{r}_{\mathrm{w}}$ radio primitivo, $\mathrm{x}_{\mathrm{pb}}$ es la distancia de contacto que varía de $-\mathrm{p}_{\mathrm{b}} / 2 \mathrm{a} \mathrm{p}_{\mathrm{b}} / 2$ en el que $\mathrm{p}_{\mathrm{b}}$ representa al módulo del engranaje, para la resolución de la expresión anterior se puede utilizar Series de Fourier.

En los 3 modelos presentados el flujo no es constante el caudal obtenido tampoco lo es, por lo que se define un coeficiente de fluctuación de caudal igual a la diferencia entre el caudal máximo y mínimo sobre el caudal medio:

$$
\eta=\frac{\dot{V}_{N, \max }-\dot{V}_{N, \min }}{\dot{V}_{N, m}}
$$


Además de modelos teóricos se han publicado modelos numéricos debido a la complejidad que representa su solución (Vacca A, 2011) presenta una simulación en HYGESim una potente herramienta para la simulación de máquinas hidráulicas de engranajes.

En los modelos expuestos se puede apreciar la importancia que tiene las características geométricas de la bomba y el tipo de perfil del diente, el presente trabajo se limita a bombas de engranajes externos con dientes de perfil evolvente por lo que para la comparación teórica experimental se utilizará el modelo propuesto por Huang debido a que considera el volumen retenido por revolución y además su modelo se adapta a bombas con y sin cámara de alivio, en el caso de un trabajo posterior que incluya bombas con dientes de perfil no evolvente se podría analizar el modelo propuesto por Nakamura que es más completo pero requiere de técnicas matemáticas más avanzadas para su análisis, el modelo de Casoli no considera el volumen retenido por revolución por lo que sería el que presente más error en comparación a los valores obtenidos experimentalmente.

Además para la adquisición de la potencia consumida hay que considerar que la potencia que una bomba de engranajes consume es difícil de predecir, la misma está en función de la calidad del fluido y suele estar afectada con la degradación del mismo (Frith R., 1996), por lo que adicional se realizará comparaciones entre bombas en estado óptimo y degradado.

\section{Metodología}

En el presente artículo se usó técnicas de investigación bibliográfica y de campo, para obtener las magnitudes necesarias para el monitoreo de sistemas oleohidráulicos. En todo proceso es absolutamente necesario controlar y mantener constantes algunas magnitudes, tales como presión, caudal, temperatura, $\mathrm{pH}$, conductividad, velocidad, humedad, punto de rocío, etcétera. Los instrumentos de medición y control permiten el mantenimiento y la regulación de estas constantes en condiciones óptimas de operación. Un transmisor recibe la señal del sensor y la transmite hacia una unidad remota. Los transmisores son instrumentos que captan la variable de proceso y la transmiten a distancia a un instrumento receptor, indicador, registrador, controlador o combinación de estos.

La mayoría de los sistemas de control para sistemas oleohidráulicos requieren la medida de presión, por lo que existen diversos tipos de sensores y medidores de presión. Para medir y monitorear la presión en el banco de pruebas didáctico utilizaremos un transductor de presión, el cual nos sirve para transformar la energía de presión en una señal electrónica. Este dispositivo será dispuesto en la línea principal de presión después de la válvula de control de la presión, el cual medirá la presión del sistema. 
El transductor de presión puede ejercer funciones como: alarma de estado de situación de una máquina, presión digital, conexión a PLC, etc. El transductor que usaremos está constituido por un cuerpo de una pieza cilíndrica de diafragma. Este elemento tiene las siguientes características:

- $\quad$ Rango de salida de señal: $20 \mathrm{~mA}$ a 5V

- $\quad$ Construcción: Cuerpo y diafragma: 17-4 PH acero inox

- $\quad$ Rango de medición: 0 - 250 Bar

El sistema de medición de temperatura de aceite en el depósito o tanque de aceite es otra variable a ser medida en el banco debido a que la viscosidad del fluido está en función de su temperatura y una temperatura elevada puede conllevar a problemas como fugas internas lo que disminuye el rendimiento del sistema. La medición y monitoreo de la temperatura se realizará con un termocupla tipo J. Una termocupla es simplemente dos alambres de distinto material unidos en un extremo. Al aplicar temperatura en la unión de los metales se genera un voltaje muy pequeño, del orden de los milivoltios el cual aumenta proporcionalmente con la temperatura. Las termocuplas $\mathrm{J}$ y $\mathrm{K}$ són económicas, físicamente muy rígidas y cubren un amplio rango de temperaturas $\left(-180\right.$ a $\left.1370{ }^{\circ} \mathrm{C}\right)$.

Para la medición del flujo de aceite hidráulico podemos usar un sensor transmisor de tecnología de inducción magnética de ruedas ovaladas que albergan imanes resistentes que giran libremente con el paso del fluido. Tienen un rango de flujo estándar 2-200 l/ min de aceite de $30 \mathrm{cSt}$ de viscosidad. El transmisor irá conectado en serie en la línea de flujo de descarga de la bomba.

\section{Características principales del transmisor de flujo:}

- $\quad$ Excelente resistencia química

- $\quad$ Construcción robusta

- $\quad$ Alta viscosidad

- $\quad$ Rango de caudal hasta 200 litros / min

- $\quad$ Rango de presión 50 - hasta 700 Bar

- $\quad$ Temperatura de trabajo de líquido $80-150^{\circ} \mathrm{C}$

- Señal de salida 4-20 mA 0-10V

\section{Configuración del banco de pruebas}

La operación del equipo se puede llevar a cabo siempre y cuando el operador, en este caso el estudiante, tenga el conocimiento de las partes principales del equipo, cuál es su funcionamiento y cuáles son las condiciones mínimas de seguridad necesarias para su operación. Todo esto con el propósito de precautelar la integridad física del operador y prolongar 
la vida útil del equipo. Con éste fin se describen los principales componentes del banco de prueba oleohidráulico, su funcionamiento y condiciones mínimas admisibles de operación. Una vez familiarizado con el banco de pruebas oleohidráulico y sumado los conocimientos teóricos adquiridos en las distintas asignaturas relacionadas con la hidráulica, el estudiante estará en condición de realizar distintos circuitos con el propósito de comprender cuál es la causa efecto que se produce al interactuar los distintos dispositivos (Gatica, 2009).

El banco de pruebas está conformado por un panel principal que soporta los componentes y accesorios en su parte superior, en la parte inferior se encuentran los componentes de accionamiento y depósito del fluido, es decir, la central oleohidráulica. La misma consta de una bomba de engranajes externos. Este equipo posee ruedas con freno para dar la posibilidad de traslado a distintas posiciones o cambio de lugar.

Algunos componentes como las válvulas se encuentran montadas en sub-bases, que a su vez cuenta con conexiones rápidas, que agiliza el armado de los circuitos. El equipo posee un motor de accionamiento monofásico que se conecta a la red eléctrica de 220 Volts. Además, el control de puesta en marcha del motor, el interruptor general y los elementos de protección se ubican en el tablero eléctrico. El estanque o depósito de aceite tipo ISO 32 con una capacidad de 15 litros. Los elementos de control y visualización se encuentran en el panel superior del banco de prueba como son: manómetros, un vacuómetro, y una pantalla Touch screen, que permiten visualizar entre algunos datos como presión, fuerza y torque. El sistema de accionamiento automático está dispuesto por medio de la botonera de control y bajo de ella se encuentra un panel de conexiones para llevar a cabo el armado de circuitos semiautomáticos o automáticos. La comunicación entre los distintos componentes, es decir entre válvulas, cilindros, motor, etc., se realiza por medio de mangueras hidráulicas que tienen en sus extremos conectores rápidos standard para todo el banco y de distintas longitudes, las mismas que se encuentran a los costados del banco de pruebas, en la figura 2 podemos ver una propuesta del diseño del banco. 
Gráfico No. 01. Esquema general Banco de Prueba Oleohidráulico

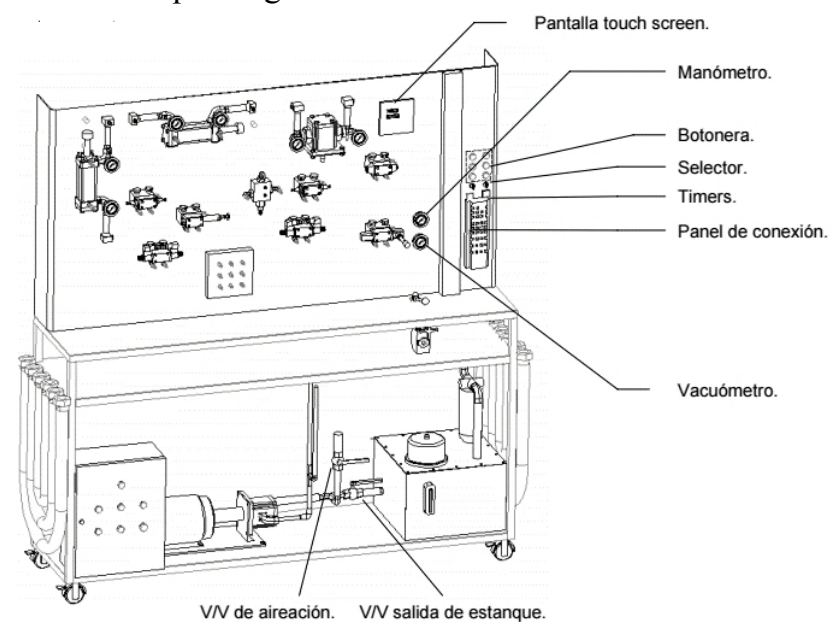

Fuente: Investigación bibliográfica, 2016

Realizado por: Los autores.

\section{Adquisición de señales del banco de pruebas}

Los sistemas de fabricación industrial, a menudo se cuentan con dos, tres o más actuadores de cilindro hidráulico sincronizados. La necesidad de diseño de un banco de pruebas (Custodio V., 2013) y un sistema de control reconfigurable que simule situaciones de la industria impulsa la investigación en el desarrollo de un sistema, conveniente para las operaciones de sistemas oleohidráulicos (Liu Hui, 2008).

El sistema de control de posición se puede subdividir en el módulo del controlador ARDUINO, y RASPBERRY subsistema electro-hidráulico y los sensores de posición. Los interruptores de límite de posición, se fijan en la plataforma de cuerpo, para detectar la posición del pistón de los cilindros hidráulicos y enviar un voltaje de señal al circuito de interconexión. El módulo del controlador de la posición se muestra en la figura, utiliza un bucle cerrado de control para sistema de control de la posición de la bobina del control direccional proporcional basado en la retroalimentación de la posición de la válvula pistón del cilindro (Gabriele A., 2015) (Chengwen W., 2013). 
Gráfico No. 02. Módulo controlador de posición

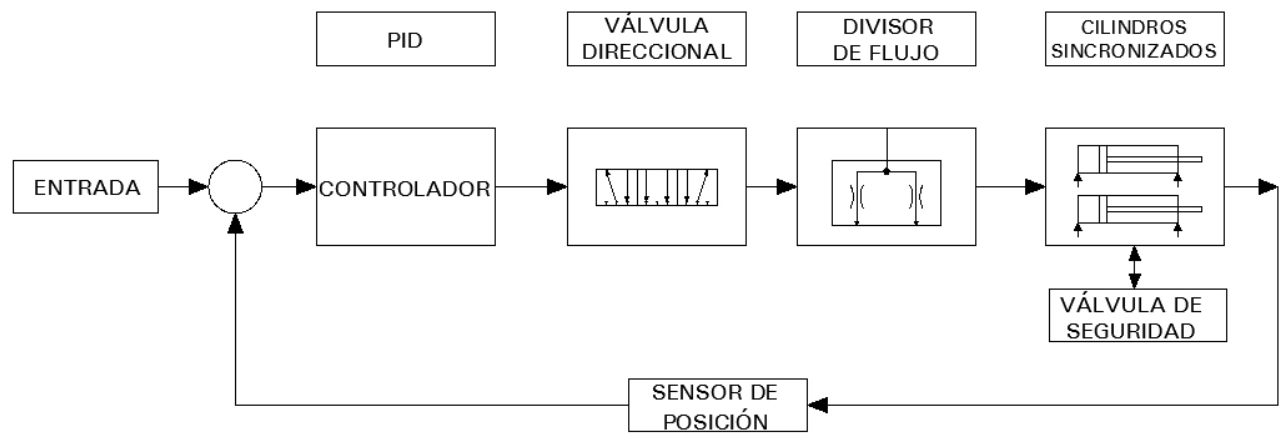

Fuente: Investigación bibliográfica, 2016

Realizado por: Los autores.

Otro método es conectar mecánicamente el actuador hidráulico es a través de cableado u otros diseños del acoplamientos. En comparación con otros métodos hidráulicos y mecánicos, electro-hidráulico de control se ofrece una alternativa flexible. Con el sistema electro-hidráulico, estrategias de control de variables se pueden diseñar para manejar diversas señales, así como incertidumbres y perturbaciones externas asociadas con los sistemas hidráulicos. En aplicaciones donde la mecanismos entre varios cilindros resultan óptimos por la exactitud del funcionamiento (Olukorede T., 2014).

Aunque un prototipo real debe ser construido, un prototipo software previamente permite agilizar procesos e identificar opciones correctas e incorrectas. Estas pruebas sin embargo pueden ir desde pequeños ensayos de funcionalidad a niveles mucho más precisos. Optimización y detalles relacionados con los controles, velocidades, pérdidas, etc., pueden ser evaluados de una manera interesante. De esta forma es posible mantener cierto nivel de innovación dentro de las prácticas de laboratorios a un costo que puede ser asumido por las instituciones educativas, permitiendo a su vez, involucrar un mayor número de estudiantes dentro de la misma infraestructura (Custodio V., 2013).

\section{Sistema de comunicación para la información remota}

En la actualidad es necesario utilizar la tecnología para facilitar el desarrollo de metodologías de aprendizaje por medio de plataformas virtuales capaces de poner al alcance de los estudiantes y docentes funciones de control y principalmente de monitoreo. Dentro de las varias propuestas sobre la adquisición de datos de múltiples variables hacia el hardware de gestión de las mismas se encuentran algunas estrategias o técnicas que aportan al mejoramiento de la calidad de la señal como por ejemplo aplicación de convertidores $\mathrm{A} / \mathrm{D}, \mathrm{D} / \mathrm{A}$, filtros, por software, entre otros. La propuesta del presente desarrollo dentro de la adquisición de datos es aplicar 
los fundamentos de la ley de ohm para a partir de una señal de corriente estándar de 4 a $20 \mathrm{~mA}$ por la utilización de instrumentación industrial y obtener el voltaje en una escala definida por el valor de una resistencia en los rangos de $300 \mathrm{ohm}$ hasta los $520 \mathrm{ohm}$ para adquirir de manera correcta las magnitudes de presión tomando en cuenta que la señal de corriente no presenta perdidas a distancias relativamente largas en el cableado para la transmisión hasta el hardware de adquisición de magnitudes como la temperatura y flujo en una tarjeta Arduino en la que se establecen las librerías para la comunicación extendida con la tarjeta Raspberry por medio de una conexión USB dentro de la cual se elevan los datos hacia el laboratorio virtual por medio de la red de internet avanzado CEDIA (Cruz, 2015).

Gráfico No. 03. Arquitectura de comunicación

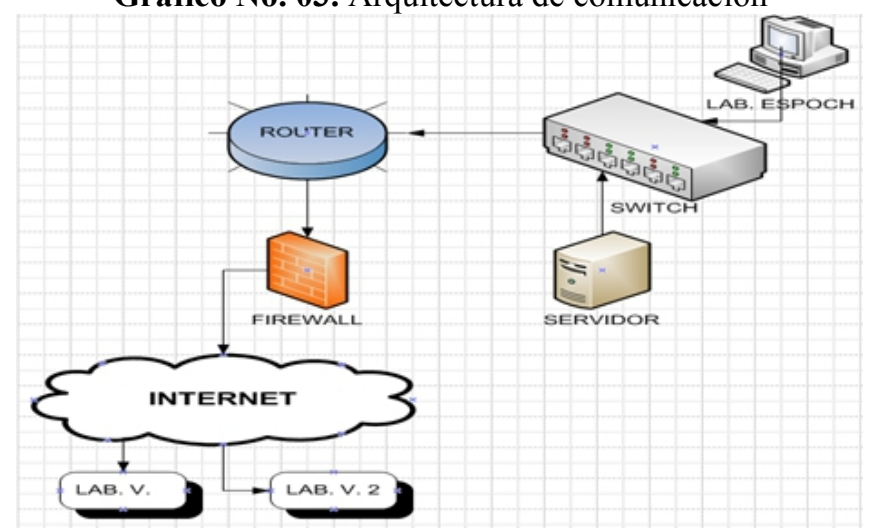

Fuente: Investigación bibliográfica, 2016

Realizado por: Los autores.

La adquisición de datos hacia la tarjeta Raspberry se la puede hacer de manera directa sin necesidad de la tarjeta Arduino, pero el inconveniente es que la adquisición de datos utiliza recursos de memoria dentro de la tarjeta Raspberry y los datos de las magnitudes se presentan con retardos. Los retardos producidos para la aplicación en un laboratorio virtual no se los considera prioritarios por que los datos se van registrando en una base de datos a la que se puede acceder y tener toda la información del proceso o practica realizada, sin embargo para algunas pruebas en las que se necesite verificar los valores mostrados en la plataforma virtual con la acción física del banco de pruebas es importante evidenciarlo casi en tiempo real, tomando en cuenta que siempre se presentará un retardo en ambos sentidos de la comunicación pero en la actualidad existen formas o métodos para bajar los tiempos de respuesta como por ejemplo la fibra óptica en la cual el ancho de banda es mucho más grande dando un excelente resultado en la entrega en la paquetería de la información. 
Gráfico No. 04. Esquema básico de laboratorio Web.

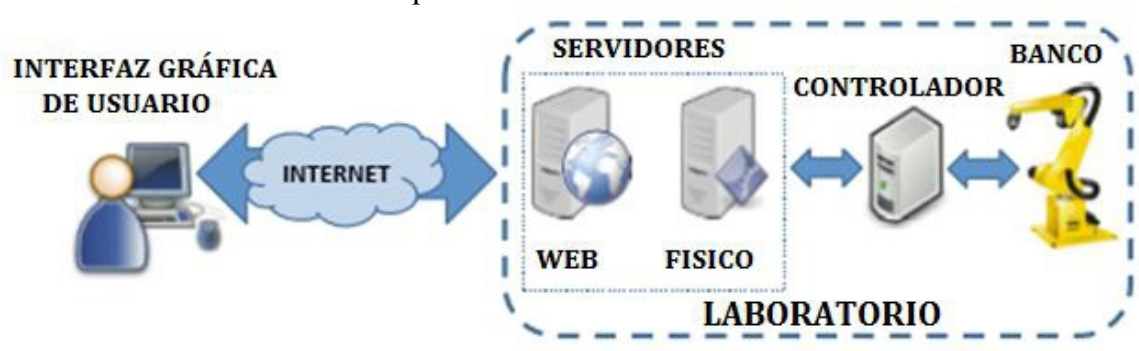

Fuente: Investigación bibliográfica, 2016

Realizado por: Los autores.

El ambiente virtual sirve para generar reportes de los resultados de ensayos o prácticas de laboratorio, una de las variables importantes para monitorear dentro de un sistema hidráulico es el fluido y las pérdidas de presión producto de fugas en las cañerías o ductos, éste desperfecto en la red hidráulica tiene que ser notificado por medios virtuales a los usuarios para tomar medidas correctivas y evitar cualquier incidente.

En base a los estudios y desarrollos realizados en el campo de laboratorios virtuales no se considera la seguridad que el banco de pruebas debe tener porque están programados solo para la tarea específica de mostrar los valores y hacer un análisis de los mismos, por lo que, en esta propuesta se plantea un sistema capaz de tomar acciones para la seguridad de los equipos y los usuarios y por medio de la programación del controlador desconecte la unidad de poder hidráulica o reducir el flujo variando la velocidad del motor por motivo de presiones elevadas no consideradas en los procesos de las prácticas de laboratorio.

Gráfico No. 05. Esquema básico de conexión.

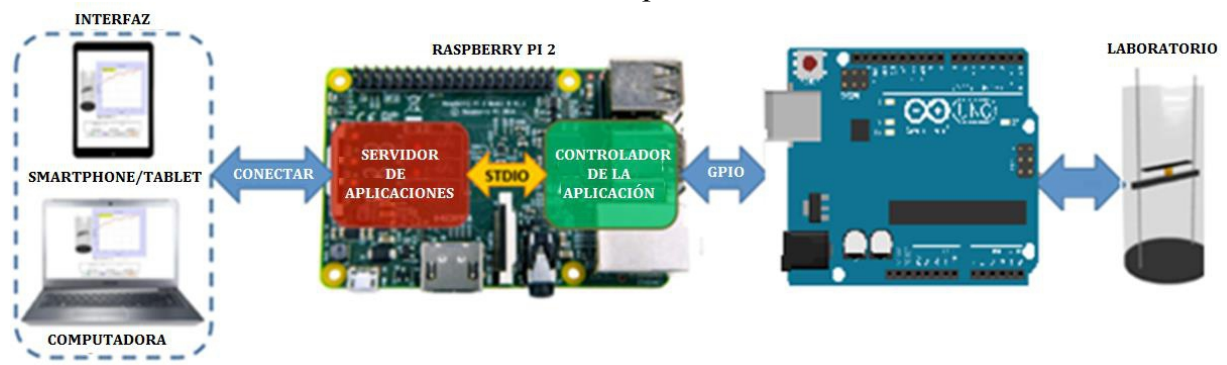

Fuente: Investigación bibliográfica, 2016

Realizado por: Los autores.

\section{Resultados esperados}

Un sistema oleohidráulico puede ser una parte esencial en una máquina para producción o trabajo pesado, la implementación de un banco de pruebas remoto para este tipo de componentes mejorará la calidad del 
aprendizaje del funcionamiento de estos sistemas en el campo de la ingeniería.

El banco de pruebas proporcionará a los estudiantes e instituciones educativas, una reducción en costos por adquisición de equipos, frecuentemente debido a que no se contaba con estos equipos muy costosos.

La Capacitación constante, sobre la operación y mantenimiento de este banco de pruebas hidráulicas, para tener siempre en buenas condiciones este equipo así como aumentar la vida útil del mismo.

Este equipo será de mucha utilidad, ya que se podrá ofrecer un servicio con un respaldo comprobable de los componentes simulados en tiempo real los componentes funcionarán de una manera óptima y el estudiante tendrá la certeza que la variable física medida es leída en tiempo real de una manera efectiva.

La educación superior se beneficiará de este tipo de laboratorios ya que ninguna universidad cuanto con una infraestructura que contemple todos los laboratorios que un estudiante necesita para su formación profesional y con la implementación de laboratorios virtuales se puede crear una red de universidades que compartan sus laboratorios para así fortalecerse entre ellas beneficiando a sus estudiantes con una formación práctica e integral.

Con el desarrollo de laboratorios virtuales los estudiantes pueden realizar varias prácticas de manera remota lo que optimiza el proceso de aprendizaje y facilita el acceso a estudiantes de diversas universidades a laboratorios remotos instalados en una red con esto el estudiante puede profundizar sus conocimientos.

\section{Conclusiones}

Es posible convertir un laboratorio normal de pruebas insitu en un laboratorio virtual remoto conectado a internet para que las pruebas puedan ser realizadas desde cualquier parte del mundo y con esto permitir al estudiante tener acceso a realizar prácticas en diversos bancos remotos y así pueda profundizar sus conocimientos.

Se ha visto la importancia de los laboratorios remotos para la enseñanza en universidades y centros de investigación debido a la poca disponibilidad de laboratorios físicos frente a la amplia capacidad que albergan las universidades hoy en día.

Se ha podido determinar las variables a ser monitoreadas en un sistema oleo hidráulico para que éste opere correctamente, como la presión la cuál debe estar limitada a la capacidad del sistema, la temperatura del fluido la cuál debe estar en un rango aceptable en función de la viscosidad de operación del aceite hidráulico para evitar fugas internas y con esto un rendimiento bajo. 
Se analizó varios modelos matemáticos que predicen el comportamiento de una bomba de engranajes externos en condiciones de vacío con la finalidad de comparar los resultados teóricos con los adquiridos en tiempo real y estimar si existe una diferencia significativa o no, además comparar los distintos modelos estudiados y determinar el modelo que más se acerca a la realidad en base a los resultados reales obtenidos en el banco de pruebas.

Para la recepción de las señales es posible incorporar una tarjeta arduino al rasberry para posteriormente convertir las señales emitidas por un banco de pruebas y enviarlas por internet a un servidor y de allí a cualquier computador para que estas señales puedan ser utilizadas en el entorno on-line del laboratorio virtual.

Con la construcción de bancos de pruebas se puede diversificar las prácticas que realizan los estudiantes en su formación profesional formando una red entre universidades, y con esto se disminuye el costo de la fabricación de laboratorios en cada universidad.ñ

\section{References:}

1. Casoli P., A. V. (2005). A numerical model for the simulation of external gear pumps. The six JFPS international symposium on fluid power. 151-160.

2. Chengwen W., Z. J. (2013). An experimental study of the dual-loop control of electro-hydraulic load simulator (EHLS). Chinese Journal of Aeronautics, 1586-1595.

3. Cruz, B. E. (2015). Remote web-based control laboratory for mobile devices based on ejss, raspberry pi and node.js. IFAC, 158-163.

4. Custodio V., A. A. (2013). Plataforma para la enseñanza a distancia de un laboratorio de neumática. SCIELO, 176-181.

5. Frith R., S. W. (1996). Comparison of an external gear pump wear model with test data. Elseiver, 64-71.

6. Gabriele A., V. A. (2015). A design solution for efficient and compact electro-hydraulic actuators. Procedia Engineering, 8-16.

7. Gatica, R. (2009). Diseño e implementación de experiencias de laboratorio en banco de prueba oleohidráulico. Universidad Austral de Chile.

8. Huang K, C. W. (2009). Kinematic flowrate characteristics of external spur gear pumps using an exact closed solution. Mechanism and Machine Theory. Vol.44, 1121-1131.

9. Lei, L. (2012). Research of the remote experiment system based on virtual. Physics Procedia 24, 1199 - 1206. 
10. Liu Hui, G. H. (2008). Application of high-speed solenoid valve to the semi-active control of landing gear. Chinese Journal of Aeronautics, 232-240.

11. Martinez P., A. N. (2014). Estado del arte sobre perfiles de dientes para engranajes. Prospect Vol.12, 29-40.

12. Nagamura K., K. I. (2004). Design and performance of gear pumps with a non-involute tooth profile. Journal of Engineering Manufacture 218, 699-710.

13. Olukorede T., K. M. (2014). Control system for electro-hydraulic synchronization on RBPT. Procedia CIRP, 835-840.

14. Petr Sládeka, L. P. (2011). Remote laboratory - new possibility for school experiments. Procedia Social and Behavioral Sciences 12, 164-167.

15. Rugeles, R. C. (2002). LSA instrumentación virtual en la enseñanza de la ingeniería electrónica. Acción pedagógica, Vol. 11, No. 1, 78 84.

16. Svishchev A., A. P. (2015). The theoretical and experimental studies comparison of the pressure pulsation in the discharge chamber of the gear pump. Procedia Engineering 113, 186 - 191.

17. Vacca A, G. M. (2011). Modelling and experimental validation of external spur gear machines for fluid power applications. Simulation Modelling Practice and Theory. Vol. 19., 2007-2031. 